\title{
Editorial: Physiology of endurance running and exercise behaviour
}

\author{
Nikolaidis, Pantelis T ; Knechtle, Beat
}

DOI: https://doi.org/10.1016/j.physbeh.2019.04.010

Posted at the Zurich Open Repository and Archive, University of Zurich ZORA URL: https://doi.org/10.5167/uzh-171213

Journal Article

Accepted Version

\section{(9) (1) $\Theta \Theta$}

The following work is licensed under a Creative Commons: Attribution-NonCommercial-NoDerivatives 4.0 International (CC BY-NC-ND 4.0) License.

Originally published at:

Nikolaidis, Pantelis T; Knechtle, Beat (2019). Editorial: Physiology of endurance running and exercise behaviour. Physiology and Behavior, 205:1.

DOI: https://doi.org/10.1016/j.physbeh.2019.04.010 
Editorial: Physiology of endurance running and exercise behaviour

Pantelis T. Nikolaidis ${ }^{1} \&$ Beat Knechtle ${ }^{2}$

${ }^{1}$ Exercise Physiology Laboratory, Nikaia, Greece

${ }^{2}$ Institute of Primary Care, University of Zurich, Zurich, Switzerland

Corresponding author

Pantelis Nikolaidis, pademil@hotmail.com 
The rationale for the present special issue was to address major Physiology \& Behavior aspects concerning the increased number of participants in outdoor endurance races during the last years (Vitti, Nikolaidis, Villiger, Onywera, \& Knechtle, 2019). Recreational marathon runners are older than 40 years old and have relatively small sport experience compared to competitive runners (Gordon et al., 2017) highlighting the need for new knowledge with regard to training and testing. In addition, since exercise is medicine (Elrick, 1996), information on the dose-response would be of great practical application.

With regards to the role of training, a comparison between training either of high-intensity or high-volume showed that both were beneficial for the endurance performance of recreational athletes (Clemente-Suarez, Delgado-Moreno, Gonzalez, Ortega, \& Ramos-Campo, 2018). It has also been supported that selfselected intensity could be an effective component of an exercise prescription (Haile, Goss, Andreacci, Nagle, \& Robertson, 2019). In addition to traditional training approach (aerobic exercise), circuit resistance training also might improve endurance (Kolahdouzi et al., 2018). It has been shown that acute carob supplementation combined with exercise training can undoubtedly improve aerobic capacities in taekwondo athletes (Gaamouri et al., 2019). Performance in endurance running relies on a series of physiological characteristics such as maximal oxygen uptake and anaerobic threshold (Sales et al., 2017). Sales and co-authors suggested that an integrative perspective of the anaerobic threshold may provide a solid background to exercise physiologists, coaches, athletes and enthusiasts to apply any of the methods of identification, since one marker seems to be dependent on the other. A submaximal 6-min exercise test protocol to assess HRC of running was considered valid to estimate $\mathrm{VVO}_{2}$ max of recreational runners both in treadmill and outdoor track (Olher et al., 2019). The beneficial role of long-term endurance exercise on cardiovascular system was confirmed (Deus et al., 2018). However, the participation in exercise is not without risks. For instance, it was shown that a mild iron deficiency might lead to decreased endurance in physically active women (Dziembowska, Kwapisz, Izdebski, \& Zekanowska, 2018). Furthermore, the acute responses of biomarkers of muscle damage and inflammation to endurance exercise might return to physiological ranges $72 \mathrm{~h}$ post-race (Rubio-Arias et al., 2018).

Of course, we are aware that the papers of this collection did not cover all the aspects that we intended to investigate. Nevertheless, we are confident that these papers provide important knowledge for the aspects they referred to and would trigger further discussion on this topic. As the phenomenon of the recreational outdoor endurance exercise continues to develop, future studies should focus to verify existed knowledge - mainly obtained in elite endurance athletes - in the specific characteristics and needs of recreational athletes.

\section{References}


Clemente-Suarez, V. J., Delgado-Moreno, R., Gonzalez, B., Ortega, J., \& RamosCampo, D. J. (2018). Amateur endurance triathletes' performance is improved independently of volume or intensity based training. Physiol Behav. doi: 10.1016/j.physbeh.2018.04.014

Deus, L. A., Sousa, C. V., Rosa, T. S., Filho, J. M. S., Santos, P. A., Barbosa, L. D., . . . Simoes, H. G. (2018). Heart rate variability in middle-aged sprint and endurance athletes. Physiol Behav. doi: 10.1016/j.physbeh.2018.10.018

Dziembowska, I., Kwapisz, J., Izdebski, P., \& Zekanowska, E. (2018). Mild iron deficiency may affect female endurance and behavior. Physiol Behav. doi: 10.1016/j.physbeh.2018.09.012

Elrick, H. (1996). Exercise is medicine. Phys Sportsmed, 24(2), 72-76. doi: 10.3810/psm.1996.02.1234

Gaamouri, N., Zouhal, H., Hammami, H., Ben Abderrahman, A., Hackney, A. C., Saeidi, A., . . Ben Ounis, O. (2019). Effects of polyphenol (carob) supplementation on body composition and aerobic capacity in taekwondo athletes. Physiol Behav.

Gordon, D., Wightman, S., Basevitch, I., Johnstone, J., Espejo-Sanchez, C., Beckford, C., ... Merzbach, V. (2017). Physiological and training characteristics of recreational marathon runners. Open Access J Sports Med, 8, 231-241. doi: 10.2147/oajsm.s141657

Haile, L., Goss, F. L., Andreacci, J. L., Nagle, E. F., \& Robertson, R. J. (2019). Affective and metabolic responses to self-selected intensity cycle exercise in young men. Physiol Behav. doi: 10.1016/j.physbeh.2019.02.012

Kolahdouzi, S., Baghadam, M., Kani-Golzar, F. A., Saeidi, A., Jabbour, G., Ayadi, A., ... Zouhal, H. (2018). Progressive circuit resistance training improves inflammatory biomarkers and insulin resistance in obese men. Physiol Behav. doi: 10.1016/j.physbeh.2018.11.033

Olher, R. R., Sales, M. M., Sousa, C. V., Sotero, R. C., Madrid, B., Cunha, R. R., . . Simoes, H. G. (2019). Heart rate cost of running in track estimates velocity associated with maximal oxygen uptake. Physiol Behav. doi: 10.1016/j.physbeh.2019.02.029

Rubio-Arias, J. A., Avila-Gandia, V., Lopez-Roman, F. J., Soto-Mendez, F., Alcaraz, P. E., \& Ramos-Campo, D. J. (2018). Muscle damage and inflammation biomarkers after two ultra-endurance mountain races of different distances: $54 \mathrm{~km}$ vs $111 \mathrm{~km}$. Physiol Behav. doi: 10.1016/j.physbeh.2018.10.002

Sales, M. M., Sousa, C. V., da Silva Aguiar, S., Knechtle, B., Nikolaidis, P. T., Alves, P. M., \& Simoes, H. G. (2017). An integrative perspective of the anaerobic threshold. Physiol Behav. doi: 10.1016/j.physbeh.2017.12.015

Vitti, A., Nikolaidis, P. T., Villiger, E., Onywera, V., \& Knechtle, B. (2019). The "New York City Marathon": participation and performance trends of 1.2M runners during half-century. Res Sports Med, 1-17. doi:

$10.1080 / 15438627.2019 .1586705$ 